\title{
Innovación y productividad en las empresas de servicios y manufactureras: el caso del Perú ${ }^{1}$
}

\author{
Mario D. Tello
}

\section{Resumen}

En este artículo se analiza la relación entre las decisiones de inversión, la intensidad de la inversión, los resultados de la innovación y la productividad del trabajo en una muestra de empresas de servicios y manufactureras del Perú en 2004, sobre la base de un modelo CDM ajustado (Crepon, Duguet y Mairesse, 1998). Algunos de los resultados obtenidos indican que el tamaño de la empresa es clave en la decisión de inversión. El tamaño de la empresa y la intensidad de la inversión también fueron determinantes fundamentales de la probabilidad de elaborar productos de innovación tecnológica y no tecnológica y de la productividad laboral de los sectores de servicios y manufacturas. Estos resultados sugieren que las políticas horizontales de ciencia, tecnología e innovación (CTI) que alientan a las empresas a incrementar la intensidad de la inversión en esas áreas pueden contribuir a aumentar la productividad del trabajo de las firmas.

\section{Palabras clave}

Sector terciario, empresas manufactureras, inversiones, ciencia y tecnología, innovaciones tecnológicas, productividad, Perú

Clasificación JEL

L8, O31

Autor

Mario D. Tello es Profesor del Departamento de Economía de la Pontificia Universidad Católica del Perú. mtello@pucp.edu.pe

\footnotetext{
1 Este artículo es una versión revisada y abreviada de un trabajo presentado en el marco del proyecto Innovation and Productivity in Services in Latin American Countries, que se completó mientras el autor se desempeñaba como Profesor Visitante del Banco de Desarrollo de América Latina (CAF) en el Centro Latinoamericano de la Universidad de Oxford. El autor agradece a Gustavo Crespi, Fernando Vargas, Diego Aboal, Esequiel Tacsir, Sylvia Dohnert y a los participantes de los seminarios realizados en el marco del proyecto por sus útiles comentarios y a Carla Solís por su excelente asistencia en la investigación.
} 


\section{Introducción}

Pese a la importancia del sector de servicios ${ }^{2}$ en muchas economías en desarrollo en términos de valor real y empleo ${ }^{3}$, los estudios del sector y sus actividades de innovación en la economía peruana son escasos. La mayor parte del trabajo en esta área se centró en el análisis de las exportaciones de servicios, en particular en el sector turístico ${ }^{4}$. Se realizaron algunos estudios descriptivos sobre la innovación, en los que se destacaba el análisis de las actividades de ciencia, tecnología e innovación (CTI), las herramientas de las tecnologías de la información y las comunicaciones (TIC) (CONCYTEC/ INEI, 2005b; Kuramoto, 2008; Tello, 2010 y 2011, entre otros) y la política económica (Comisión Consultiva para la Ciencia, Tecnología e Innovación, 2012; Kuramoto y Díaz, 2010 y 2011; Kuramoto, 2007; Tello, 2010; Sagasti, 2011, y UNCTAD/CEPAL, 2011, entre otros).

En ese sentido, y sobre la base de una encuesta acerca de las actividades de CTI a nivel empresarial realizada por el Consejo Nacional de Ciencia, Tecnología e Innovación Tecnológica (CONCYTEC) y el Instituto Nacional de Estadística e Informática (INEI), el principal aporte de este trabajo consiste en analizar por primera vez la relación entre la inversión en CTI, los resultados de la innovación y la productividad de las empresas del sector de servicios del Perú en 2004 mediante un modelo de Crepon, Duguet y Mairesse (CMD) (1998) estándar ajustado. Para fines comparativos, se analiza también la misma relación en las empresas del sector manufacturero.

El trabajo se divide en seis secciones, incluida esta Introducción. En la segunda sección se presenta una breve reseña de la literatura y en la tercera se describe la muestra de datos tomada de la Encuesta Nacional de Ciencia, Tecnología e Innovación Tecnológica de 2004 (ENCYT-04) (INEI, 2005). En la cuarta sección se formula el modelo estructural de innovación y productividad y en la quinta se presentan los resultados de la estimación. En la sexta y última sección se detallan las principales conclusiones del trabajo y se realizan algunas reflexiones sobre las políticas de fomento de las actividades de CTI.

\section{Breve reseña de la literatura}

La literatura sobre el proceso de innovación, sus limitaciones y sus efectos en el desempeño de las empresas en América Latina surgió en las dos últimas décadas a partir de las encuestas sobre CTI y TIC realizadas a nivel empresarial ${ }^{5}$. En Mairesse y Sassenou (1991), OCDE (2009), Mairesse y Mohnen (2010), Crespi y Zúñiga (2010) y Hall (2011) se hace referencia a los estudios relativos a los países desarrollados y a algunas economías en desarrollo 6 . La metodología que predomina en la mayoría de ellos es la propuesta por Crepon, Duguet y Mairesse (1998), conocida como modelo CDM ${ }^{7}$. Este modelo presenta dos características principales: la especificación de un modelo estructural en el

2 Este sector incluye electricidad y agua, servicios financieros y de seguros, servicios gubernamentales, servicios para hogares y empresas, transporte, telecomunicaciones, servicios de educación y salud privados y hoteles y restaurantes.

3 De acuerdo con el Banco Mundial (2013), el sector de servicios representaba, en promedio, el 59\% del producto interno bruto (PIB) en 2000-2010 y el 77\% del empleo formal total en 2000-2009.

4 Las exportaciones de servicios representan alrededor del 3\% del PIB. Esto significa que los servicios en el Perú constituyen sobre todo una industria orientada al mercado interno (Tello, 2012a).

5 Por ejemplo, Crespi y Zúñiga (2010) utilizan seis conjuntos de datos de encuestas sobre CTI realizadas en la Argentina (que cubren el período 1998-2001), Chile (2004-2005), Colombia (2004), el Uruguay (2006) y Costa Rica (2008) para analizar los efectos de la innovación en la productividad de las empresas. Balboni, Rovira y Vergara (2011) utilizan las encuestas sobre TIC para analizar los efectos de esas tecnologías en el desempeño de las empresas manufactureras en la Argentina, Chile, Colombia, el Perú y el Uruguay.

6 Los estudios de Griliches (1979) y Griliches y Pakes (1980) son pioneros en esta materia.

7 La metodología alternativa se basa en estimaciones de la productividad total de los factores o productividad del trabajo mediante datos de panel, datos de corte transversal o ambos. 
que variables como los gastos en investigación y desarrollo $(l+D)$, los productos de innovación y la productividad (del trabajo) de las empresas están interrelacionadas y el uso de técnicas econométricas para abordar los sesgos de selección y simultaneidad y algunas características estadísticas de los datos disponibles.

De acuerdo con Crespi y Zúñiga (2010), los modelos CDM comprenden cuatro etapas: la decisión de una empresa de invertir en actividades de innovación ${ }^{8}$, las decisiones de la empresa sobre el monto de la inversión, la producción de conocimiento tecnológico y no tecnológico como resultado de esa inversión y el efecto de la innovación en la productividad. Esos autores también hacen una lista de los resultados empíricos relevantes con respecto a los factores incluidos en esas cuatro etapas. En primer lugar, el tamaño de la empresa, la participación de mercado y la diversificación de productos parecen aumentar la probabilidad de invertir en actividades de CTI. La fuerza resultante del incremento de la demanda y la tecnología también aumentan dicha probabilidad. En segundo lugar, la productividad de una empresa parece estar positivamente correlacionada con la producción de innovación, incluso después de controlar la calificación de la mano de obra. En tercer lugar, la innovación tecnológica (en productos o procesos) puede conducir a un aumento de la productividad, las ventas y las ganancias de las empresas (véase en Mohnen y Röller (2005) esta conclusión con respecto a empresas europeas). Por último, las empresas que invierten de forma más intensiva en I+D son más propensas a desarrollar innovaciones - de productos, procesos o patentes-, después de corregir la endogeneidad y controlar características de la empresa como tamaño, afiliación a un grupo o tipo de estrategias de innovación aplicadas (externalización y colaboración en l+D, entre otras).

No obstante, la evidencia con respecto a la capacidad de las empresas de los países en desarrollo para transformar la I+D en innovación es menos clara que en el caso de las empresas de las economías industrializadas. Los resultados sobre el impacto de la innovación en la productividad del trabajo en las empresas latinoamericanas no son concluyentes. La falta de una correlación significativa entre la I+D y los resultados de la innovación y la productividad en los países en desarrollo podría explicarse porque las empresas de esos países están demasiado lejos de la frontera tecnológica y los incentivos para invertir en innovación son débiles o inexistentes. En muchas economías latinoamericanas, las innovaciones de las empresas consisten básicamente en cambios progresivos con poco o ningún impacto en los mercados internacionales y se basan sobre todo en la imitación y la transferencia de tecnología, como la adquisición de maquinaria y equipo y la compra de tecnología no incorporada. La inversión en I+D es en muchos casos prohibitiva (tanto en términos de costos financieros como de capital humano necesario) y, debido a los efectos acumulativos, podría requerir horizontes de tiempo más largos para producir resultados ${ }^{9}$.

Además de las características de las empresas, los modelos CDM incluyen fuerzas externas que influyen simultáneamente en las decisiones de innovación de los empresarios. Tradicionalmente, estas corresponden a indicadores de innovación impulsada por la demanda (normas ambientales, en materia de salud o seguridad, entre otras), impulso tecnológico (oportunidades científicas, entre otras), política de innovación (incluidos los subsidios a las actividades de l+D) y efectos indirectos.

8 De acuerdo con los datos de la encuesta disponibles para el Perú, en lugar de la inversión en I+D en este trabajo se utiliza la inversión en ciencia, tecnología e innovación (CTI). Esta incluye los gastos en actividades de ciencia y tecnología (CT) (como investigación y desarrollo experimental, formación de recursos humanos en ciencia y tecnología y servicios científicos y tecnológicos) y en actividades de innovación (como investigación y desarrollo, inversión de capital, diseño de hardware y software para producir innovación en productos, procesos, organización y comercialización). Las actividades de CT se relacionan con la generación, producción, divulgación y aplicación de conocimientos científicos y técnicos en todos los campos de la ciencia y la tecnología. Las actividades de innovación son medidas tomadas por las empresas con el objetivo de implementar nuevos conceptos, ideas y métodos para adquirir, asimilar o incorporar nuevos conocimientos.

9 Raffo, Lhuillery y Miotti (2008) realizaron un estudio comparativo de la innovación en empresas de países de América Latina (Argentina, Brasil y México) y Europa (España, Francia y Suiza). Además de diferencias estructurales entre Europa y América Latina, esos autores encontraron heterogeneidad dentro de cada región. En particular, si bien las empresas tienden a realizar actividades de innovación para lograr un mejor rendimiento económico de manera similar, su interacción con los sistemas nacionales es más débil en los países en desarrollo. Asimismo, las filiales de las empresas multinacionales extranjeras tienen un efecto heterogéneo en la innovación, que conduce a un incremento de la productividad en todos los países. 
Un aspecto señalado por Álvarez y Crespi (2011) se refiere a las limitaciones financieras. Que las restricciones de crédito podrían afectar gravemente a la innovación es una conjetura de larga data en el campo de la economía de la innovación. La innovación es el resultado de inversiones en conocimiento y este presenta por lo menos cuatro atributos específicos que pueden tener grandes repercusiones en el financiamiento de la innovación. El primero es su carácter de bien semipúblico, que impide a las empresas innovadoras excluir a otros del uso de las innovaciones que crean. Este atributo puede explicar no solo por qué las empresas invierten de manera insuficiente en innovación, sino también los límites a su financiamiento. El segundo atributo es que las inversiones en conocimiento producen un activo intangible - vinculado al capital humano que trabaja en la empresa (por ejemplo ingenieros y técnicos) -, que puede ser muy difícil de utilizar como garantía prendaria. Los bancos prefieren activos físicos para garantizar los préstamos y pueden mostrarse reacios a prestar cuando el proyecto supone la acumulación de activos intangibles, parcialmente incorporados en el capital humano de los empleados de la empresa, que pueden perderse si estos dejan la organización. El tercer atributo es que las inversiones en conocimiento tienen componentes tácitos que son idiosincrásicos de una empresa. Eso significa que una parte posiblemente sustancial de las inversiones en CTI está sumergida y no puede desplegarse fácilmente en otras actividades. El cuarto atributo es la incertidumbre asociada con sus resultados. La incertidumbre en este caso se relaciona con la falta de una distribución bien definida de la probabilidad de posibles efectos. En este contexto, las inversiones en conocimiento se parecen a las opciones, en la medida en que puede valer la pena emprender algunos proyectos con muy pocas probabilidades de éxito incluso aunque no pasen un análisis costo-beneficio ex ante. Todos estos atributos pueden tener importantes efectos en el financiamiento de la innovación.

Otro aspecto importante señalado en la literatura sobre innovación y productividad es la especificidad de las actividades productivas analizadas. Según Tacsir y otros (2011), los servicios no se consideran actividades muy predispuestas para la innovación y los encargados de la formulación de políticas de las economías en desarrollo no suelen pensar en ellos como un área estratégica en la búsqueda de un crecimiento sostenible. Eso no ocurre en las economías desarrolladas, donde se reconoce cada vez más el papel del sector de servicios como un poderoso impulsor del crecimiento y uno de los principales proveedores de empleo (Gallouj y Weinstein, 1997; Gallouj, 2002; Evangelista y Savona, 2003; Cainelli, Evangelista y Savona, 2006; Crespi y otros, 2006; Gallouj y Savona, 2009; Gallouj y Djellal, 2008 y 2010; Comisión Europea, 2011).

Gallouj y Savona (2009), Gallouj y Djellal (2008 y 2010) y Mothe y Nguyen Thi (2010), entre otros, realizan un análisis teórico, conceptual y empírico de la innovación en los servicios en los países desarrollados. En su enfoque teórico y conceptual, Gallouj y Savona (2009) y Mothe y Nguyen Thi (2010) distinguen entre un enfoque tecnológico o de asimilación (según el cual la innovación en los servicios consiste en la adopción y el uso de tecnología o los servicios son semejantes a las manufacturas), un enfoque de demarcación o diferenciación orientado a los servicios (que destaca las especificidades del producto y los procesos de producción de los servicios, por lo cual la innovación en los servicios requiere teorías específicas) y un enfoque integrador o sintetizador (en el que la innovación puede ocurrir tanto en los servicios como en las manufacturas y, dada la propensión a la convergencia entre los productos manufacturados y los servicios, apoya el desarrollo de un marco conceptual común). En este sentido, el modelo CDM aplicado a los servicios parece estar más alineado con el enfoque sintetizador.

En el caso de la literatura empírica sobre la innovación en los servicios (Gallouj y Djellal, 2010; Carayannis, Varblane y Roolaht, 2012), los estudios indican que, en primer lugar, la I+D desempeña solo un papel marginal en algunos servicios y que las empresas de servicios rara vez utilizan patentes para proteger sus productos innovadores de las imitaciones. En segundo lugar, muchos sectores que dependen en gran medida de los gastos en TIC son los más innovadores después de los sectores basados en la ciencia. Estos sectores cooperan activamente con industrias y empresas clientes posicionadas en las fases finales de la cadena de valor (servicios financieros y de venta al por menor). 
En tercer lugar, los sectores de servicios más tradicionales (servicios públicos) incluyen un conjunto de innovadores mediocres que procuran introducir tecnologías de hardware racionalizadas y de reducción de gastos, que solo involucran TIC en pequeña medida. En cuarto lugar, las características de los productos y los procesos de producción y prestación de servicios (así como el tamaño de las empresas), en términos de grado de estandarización, fueron las principales variables que afectaron la propensión a innovar y el tipo de innovación realizada. En quinto lugar, las empresas de servicios tienden a cooperar y establecer "modalidades abiertas" de innovación y se basan en competencias de alto nivel y en un tipo particular de capital humano, es decir, tienden a surgir de las disciplinas humanísticas y las ciencias blandas. Por último, pese a la gran heterogeneidad sectorial y empresarial en el sector de servicios, la innovación desempeña un papel significativo en los incrementos de productividad a nivel empresarial.

Los resultados de estudios comparativos sobre la innovación en los sectores de servicios y manufacturero presentados en Tether (2005), Rubalcaba, Gago y Gallego (2010) y Masso y Vahter (2011) indican que, si bien las empresas que realizan actividades de servicios de hecho innovan, no está claro si la intensidad de dicha innovación es mayor o menor que en las empresas manufactureras. A diferencia de lo que ocurre en estas últimas, la innovación en los servicios parece estar orientada a promover el cambio organizacional, utilizar la colaboración con clientes y proveedores, adquirir propiedad intelectual externa y hacer hincapié en la capacidad y el profesionalismo de sus trabajadores. La innovación es frecuente en los servicios intensivos en conocimiento y la innovación de productos presenta una marcada correlación con una mayor productividad. Al igual que en el sector manufacturero, el principal determinante de la innovación en los servicios es el conocimiento formal que deriva de las actividades de I+D o de la adquisición de equipos, patentes o licencias. Sin embargo, el aporte medio de la I+D al proceso de innovación tiende a ser mucho menor en el sector de servicios en su conjunto que en el sector manufacturero. Esto puede deberse a que en los servicios la I+D a menudo se realiza de manera más informal.

Estos estudios parecen concordar en que, no obstante las grandes disparidades entre los bienes y los servicios, las diferencias entre esos dos sectores son, en cierta medida, menores que las diferencias entre algunos pares de sectores de servicios, debidas a la heterogeneidad de las actividades de servicios.

Los aspectos de la innovación que derivan de la interacción entre clientes y proveedores están ganando terreno en todos los sectores económicos (incluidos los de servicios y manufacturero). La evidencia en países europeos muestra que los clientes pueden desempeñar un papel muy significativo en el impacto de calidad de los servicios, a diferencia de lo que ocurre en las industrias de bienes. Esta creciente integración entre los bienes y los servicios abre la puerta a una interpretación de los estudios y las políticas relacionadas con la innovación en los servicios que tiene en cuenta las peculiaridades, va más allá de las diferencias y se concentra en los aspectos comunes a todas las actividades productivas. Así, las medidas de política horizontales también pueden utilizarse para promover una política orientada a los servicios, basada en el tratamiento de la innovación en ese sector como una dimensión sistémica de cualquier sistema innovador.

A diferencia de los estudios sobre las economías avanzadas, la literatura y la evidencia empírica sobre la innovación en los servicios en los países latinoamericanos son limitadas. En la literatura disponible se indica la creciente importancia de la innovación en los servicios (por ejemplo, Garrido, 2009) y se constata que las empresas de servicios latinoamericanas de hecho innovan, a veces incluso más que sus pares del sector manufacturero. Las empresas de la región a menudo enfrentan gravosas limitaciones financieras al tratar de innovar, que en algunos casos pueden ser más vinculantes en el sector de servicios que en el manufacturero (Llisteri y García-Alba, 2008). Con este trabajo se procura llenar un vacío en la literatura mediante el análisis de la interrelación entre innovación y productividad en las empresas de servicios y manufactureras del Perú, sobre la base de dos modelos CDM que se presentan en la próxima sección. 


\section{Descripción de los datos}

La principal fuente de datos a nivel empresarial utilizada en este artículo es la Encuesta Nacional de Ciencia, Tecnología e Innovación Tecnológica (ENCYT-04) realizada por el CONCYTEC y el INEl entre octubre y noviembre de 2004 (CONCYTEC/INEI, 2005a) ${ }^{10}$. La ENCYT-04 proporciona información sobre las actividades de ciencia, tecnología e innovación tecnológica de 4.898 empresas de 44 sectores de la Clasificación Industrial Internacional Uniforme de Todas las Actividades Económicas (CIIU Rev. 3). En el cuadro 1 se resume un conjunto de indicadores clave para una muestra de 3.888 empresas en los sectores de servicios (2.732) y manufacturero (1.156).

La estimación del modelo CDM se basa en las cifras que se detallan en el cuadro 1. Las empresas del sector de servicios se dividen en servicios intensivos en conocimiento y servicios tradicionales y las empresas manufactureras en empresas de alta tecnología y de baja tecnología ${ }^{11}$.

Cuadro 1

Perú: indicadores de ciencia, tecnología e innovación (CTI) por tipo de empresa, en relación con todas las empresas, 2004

(En porcentajes)

\begin{tabular}{|c|c|c|c|c|c|c|c|c|c|c|}
\hline \multirow[b]{2}{*}{ Industria } & \multicolumn{5}{|c|}{ Servicios } & \multicolumn{5}{|c|}{ Manufacturera } \\
\hline & Total & $\begin{array}{l}\text { Intensivos en } \\
\text { conocimiento }\end{array}$ & Tradicionales & $\begin{array}{c}\text { Propiedad } \\
\text { nacional }\end{array}$ & $\begin{array}{l}\text { Propiedad } \\
\text { extranjera }\end{array}$ & Total & $\begin{array}{c}\text { Baja } \\
\text { tecnología }\end{array}$ & $\begin{array}{c}\text { Alta } \\
\text { tecnología }\end{array}$ & $\begin{array}{l}\text { Propiedad } \\
\text { nacional }^{\mathrm{a}}\end{array}$ & $\begin{array}{l}\text { Propiedad } \\
\text { extranjera }^{\mathrm{a}}\end{array}$ \\
\hline \multicolumn{11}{|c|}{ I. Indicadores de producción } \\
\hline $\begin{array}{l}\text { Número de } \\
\text { empresas }\end{array}$ & 2732 & 738 & 1994 & 2592 & 140 & 1156 & 954 & 202 & 1071 & 85 \\
\hline \multicolumn{11}{|c|}{ Innovación tecnológica } \\
\hline Prod. & 12,8 & 17,2 & 11,1 & 11,5 & 35,7 & 24,6 & 23,1 & 31,7 & 25,6 & 49,0 \\
\hline Proc. & 13,1 & 16,1 & 11,9 & 12,1 & 31,4 & 26,0 & 24,3 & 33,7 & 26,3 & 49,0 \\
\hline $\operatorname{lnn} .^{{ }^{b}}$ & 18,0 & 23,0 & 16,1 & 16,6 & 44,3 & 32,9 & 30,8 & 42,6 & 34,5 & 59,6 \\
\hline Internac & 10,0 & 14,2 & 8,4 & 8,9 & 30,0 & 19,6 & 17,9 & 27,7 & 20,3 & 40,4 \\
\hline Nueva $^{d}$ & 4,7 & 6,4 & 4,0 & 4,4 & 8,6 & 9,4 & 8,9 & 11,9 & 10,0 & 15,4 \\
\hline \multicolumn{11}{|c|}{ Innovación no tecnológica } \\
\hline Org. & 19,6 & 20,6 & 19,3 & 19,1 & 30,0 & 22,5 & 20,1 & 33,7 & 22,5 & 43,3 \\
\hline Com. & 14,4 & 13,4 & 14,7 & 14,1 & 19,3 & 15,2 & 13,5 & 23,3 & 15,0 & 33,7 \\
\hline Org. 0 com. ${ }^{\mathrm{e}}$ & 23,2 & 23,4 & 23,1 & 22,6 & 33,6 & 25,8 & 23,6 & 36,1 & 25,8 & 52,9 \\
\hline Tec. o no tec. ${ }^{\dagger}$ & 28,1 & 31,3 & 26,9 & 26,9 & 50,7 & 38,2 & 35,7 & 49,5 & 39,9 & 68,3 \\
\hline Tec. y no tec. ${ }^{9}$ & 13,1 & 15,2 & 12,3 & 12,3 & 27,1 & 20,5 & 18,7 & 29,2 & 20,3 & 44,2 \\
\hline
\end{tabular}

\footnotetext{
${ }^{10}$ Estas instituciones condujeron recientemente una encuesta similar a la ENCYT-04 para recoger datos relativos a 2012, pero solo sobre las empresas manufactureras.

${ }^{11}$ Los servicios intensivos en conocimiento incluyen el código 6 de la CllU, relativo a actividades como el transporte (por vía terrestre, acuática y aérea), actividades de transporte auxiliares, postales y de telecomunicaciones, intermediación financiera y actividades de seguros y fondos de pensiones, así como el código 7, que incluye actividades informáticas y relacionadas, investigación y desarrollo y otras actividades empresariales. Los servicios tradicionales incluyen las ramas de la CIIU de los códigos 3 a 9, por ejemplo reciclado, electricidad, agua, comercio al por mayor y al por menor, hoteles y restaurantes, actividades inmobiliarias, alquiler de maquinaria, salud y asistencia social, depuración de aguas residuales y gestión de desechos, actividades de asociaciones y recreativas. Las empresas manufactureras de alta tecnología incluyen los códigos 2 y 3 de la CIIU, por ejemplo productos químicos, maquinaria y equipo, maquinaria eléctrica, equipo de comunicaciones, instrumentos médicos y de precisión, y vehículos y otro equipo de transporte. Las empresas manufactureras de baja tecnología incluyen los códigos 1, 2 y 3 de la CIIU, relativos a productos alimenticios, bebidas, productos de tabaco, textiles, prendas de vestir, cuero y calzado, madera, papel, reproducción de grabaciones, productos de la refinación del petróleo, caucho, productos minerales no metálicos, metales comunes, otro equipo de transporte, productos elaborados de metal y muebles.
} 
Cuadro 1 (conclusión)

\begin{tabular}{|c|c|c|c|c|c|c|c|c|c|c|}
\hline \multirow[b]{2}{*}{ Industria } & \multicolumn{5}{|c|}{ Servicios } & \multicolumn{5}{|c|}{ Manufacturera } \\
\hline & Total & $\begin{array}{l}\text { Intensivos en } \\
\text { conocimiento }\end{array}$ & Tradicionales & $\begin{array}{c}\text { Propiedad } \\
\text { nacional }\end{array}$ & $\begin{array}{l}\text { Propiedad } \\
\text { extranjera }\end{array}$ & Total & $\begin{array}{c}\text { Baja } \\
\text { tecnología }\end{array}$ & $\begin{array}{c}\text { Alta } \\
\text { tecnología }\end{array}$ & $\begin{array}{l}\text { Propiedad } \\
\text { nacional }^{\mathrm{a}}\end{array}$ & $\begin{array}{l}\text { Propiedad } \\
\text { extranjera }^{2}\end{array}$ \\
\hline \multicolumn{11}{|c|}{ II. Indicadores de insumos } \\
\hline Gastos CTI. ${ }^{\text {h }}$ & 4,9 & 9,4 & 2,9 & 5,2 & 2,1 & 4,4 & 4,3 & 5,0 & 4,7 & 2,5 \\
\hline $1+D^{i}$ & 2,6 & 3,3 & 2,2 & 2,5 & 3,0 & 10,2 & 8,6 & 16,5 & 8,9 & 20,4 \\
\hline Сар. Сті & 19,9 & 16,9 & 21,2 & 20,8 & 11,2 & 28,8 & 31,0 & 22,8 & 30,1 & 23,3 \\
\hline 0. CT/k & 77,5 & 79,8 & 76,5 & 76,7 & 85,8 & 61,0 & 60,4 & 61,0 & 61,1 & 56,7 \\
\hline Emp. I+D' & 5,3 & 7,1 & 4,5 & 5,1 & 6,6 & 18,3 & 15,4 & 29,7 & 16,5 & 32,8 \\
\hline Emp. I+D 3 años ${ }^{m}$ & 9,6 & 11,3 & 8,9 & 9,1 & 18,6 & 14,8 & 12,9 & 23,8 & 13,7 & 28,2 \\
\hline \multicolumn{11}{|c|}{ III. Indicadores de política } \\
\hline M. int. ${ }^{n}$ & 1,6 & 2,0 & 1,5 & 1,4 & 5,0 & 4,8 & 4,6 & 5,5 & 4,3 & 10,6 \\
\hline Coop. ${ }^{0}$ & 3,7 & 4,2 & 3,6 & 3,6 & 7,1 & 6,6 & 6,3 & 5,0 & 5,8 & 9,4 \\
\hline $\mathrm{Co}-\mathrm{U} / \mathrm{G}^{p}$ & 2,1 & 2,6 & 1,9 & 1,9 & 4,3 & 3,8 & 3,9 & 3,5 & 3,7 & 4,7 \\
\hline Ap. púb.q & 2,2 & 3,1 & 1,8 & 1,9 & 7,1 & 7,9 & 7,9 & 15,4 & 6,5 & 24,7 \\
\hline Patent. $^{r}$ & 1,2 & 0,8 & 1,4 & 1,0 & 4,3 & 3,9 & 3,9 & 6,9 & 3,8 & 4,7 \\
\hline
\end{tabular}

Fuente: Elaboración propia, sobre la base de Consejo Nacional de Ciencia, Tecnología e Innovación Tecnológica/Instituto Nacional de Estadística e Informática (CONCYTEC/INEI), Encuesta Nacional de Ciencia, Tecnología e Innovación Tecnológica (ENCYT-04), Lima, 2005.

a La muestra de los indicadores de producción para las empresas nacionales y extranjeras fue de 1.196 y 104 empresas, respectivamente.

b Innovación de productos o procesos.

c Estas empresas produjeron innovación de cualquier tipo (productos, procesos, comercialización y organización) con fondos propios y sin la colaboración de otras entidades.

d Nueva en innovación de productos en el mercado.

e Innovación de organización o comercialización.

f Innovación tecnológica o no tecnológica.

g Innovación tecnológica y no tecnológica.

h Gastos totales en CTI (como porcentaje del volumen de negocios total).

i Gastos en I+D como porcentaje del gasto total en CTI.

j Gastos en capital de CTI como porcentaje del gasto total en CTI.

k Gastos en otras actividades de CTI como porcentaje del gasto total en CTI. Estas actividades de CTI incluyen formación, servicios de consultoría, ingeniería y diseño industrial, software y servicios tecnológicos.

I Empresas que invirtieron en I+D.

$m$ Empresas que invirtieron en $\mathrm{I}+\mathrm{D}$ en cualquier momento en los últimos tres años.

n Proporción de empresas activas en los mercados internacionales.

- Proporción de empresas que cooperaron en actividades de innovación.

p Proporción de empresas que cooperaron con universidades, institutos de educación superior o institutos de investigación gubernamentales.

q Proporción de empresas que recibieron apoyo financiero público para innovación.

$r$ Proporción de empresas que solicitaron una o más patentes.

El valor agregado real de estas empresas ${ }^{12}$ (en precios de 1994) representó el 31,9\% del valor agregado total de ambos sectores en 2004: el 33,3\% en el sector de servicios y el $27,4 \%$ en el manufacturero ${ }^{13}$. Las principales características de las cifras del cuadro 1 son las siguientes: en

12 El valor agregado de las empresas proviene de los datos de ventas. Estos se obtuvieron mediante el cociente entre el valor agregado y el valor de producción del respectivo sector de la CIIU tomado de los cuadros de insumo-producto de 1994 y 2007 proporcionados en forma preliminar por el INEI.

13 Para los cuatro grupos de la CIIU, la proporción de valor real de las empresas en relación con el respectivo valor real del universo fue del $21,19 \%$ en los servicios intensivos en conocimiento, el 43,79\% en los servicios tradicionales, el 22,44\% en las empresas de alta tecnología y el $27,37 \%$ en las empresas de baja tecnología. En el caso del empleo formal, las cifras son las siguientes: el $12,53 \%$ en los servicios intensivos en conocimiento, el $18,87 \%$ en los servicios tradicionales, el $24,25 \%$ en las empresas manufactureras de baja tecnología y el $23,56 \%$ en las empresas manufactureras de alta tecnología. 
primer lugar, la proporción de empresas que innovan (ya sea innovación tecnológica, no tecnológica o ambas) era mayor entre las empresas manufactureras $(38,2 \%)$ que en las de servicios $(28,1 \%)$. Sin embargo, las empresas de servicios presentaban una proporción mayor de innovación no tecnológica que de innovación tecnológica, mientras que en las empresas manufactureras ocurría lo contrario.

La proporción de empresas de propiedad extranjera (aquellas con más del 10\% de capital extranjero) que innovan fue mayor que la de las empresas de propiedad nacional en ambos sectores. En segundo lugar, la intensidad de la inversión de las empresas (medida como proporción del gasto en actividades de CTI con respecto a las ventas totales) fue ligeramente superior en el sector de servicios $(4,9 \%)$ que en el manufacturero $(4,4 \%)$. Los porcentajes más altos se registraron en las empresas de servicios intensivos en conocimiento y manufactureras de alta tecnología (el 9,4\% y el 5\%, respectivamente). Además, los índices de inversión en CTI de las empresas nacionales fueron más altos que los de las empresas extranjeras, tanto en el sector de servicios como en el manufacturero. Por otra parte, la proporción de empresas que innovaron de manera constante en el sector manufacturero $(14,8 \%)$ fue mayor que en el sector de servicios $(9,6 \%)$. Más del $50 \%$ de los gastos en CTI en ambos sectores se destinó a actividades relacionadas con formación, servicios de consultoría, ingeniería y diseño industrial, software y servicios tecnológicos.

En tercer lugar, y desde el punto de vista de la política, las empresas en general no colaboraron con otras entidades para fines de innovación. En todo caso, la proporción de empresas manufactureras que sí colaboraron $(6,1 \%)$ fue superior a la respectiva proporción de empresas de servicios $(3,7 \%)$. Las cifras relativas a la exposición internacional de las empresas también resultaron bajas y una vez más la proporción correspondiente al sector manufacturero $(4,8 \%)$ fue mayor que la del sector de servicios $(1,6 \%)$. Se obtuvieron cifras similares con respecto a la posesión de patentes. Una mayor proporción de empresas extranjeras colaboraron con respecto a las solicitudes de patentes y a la exposición internacional en ambos sectores, en comparación con las empresas nacionales. Por último, el porcentaje de empresas que reciben apoyo financiero público para actividades de innovación fue mayor en el sector manufacturero (7,9\%) que en el de servicios (2,2\%). De manera inesperada, la proporción de empresas extranjeras que reciben apoyo financiero público superó la respectiva proporción de empresas nacionales en ambos sectores.

En resumen, las cifras de 2004 sobre las actividades de CTI de las empresas concuerdan con los bajos indicadores de inversión en CTI registrados en el Perú, incluso en 2012. Aunque un tercio de las empresas de la muestra no realizó actividades de CTI, la cifra media destinada a esas actividades por trabajador en ambos sectores fue de 2.353 dólares (en dólares constantes de 1994), que equivale a menos de 6,5 dólares por día por trabajador. La medida en que esta pequeña suma afecta el desempeño de las empresas (incluida la productividad del trabajo) se investiga en las secciones siguientes.

\section{Modelos CDM y estrategia de estimación}

El modelo CDM que ha de estimarse tiene las siguientes ecuaciones:

$$
\begin{aligned}
& \mathrm{ID}_{i}^{*}=\mathrm{X}_{1 i} \cdot \beta_{1}+\alpha_{1} \cdot \mathrm{FC}_{i}+\varepsilon_{1 i} \text {; donde si } \mathrm{ID}_{i}^{*}>\mu_{i} \text { entonces } \mathrm{D}_{I D i}=1 \text {; de lo contrario } \mathrm{D}_{I D i}=0 \text {; } \\
& \mathrm{IE}_{i}^{*}=\mathrm{X}_{2 i} \cdot \beta_{2}+\alpha_{2} \cdot \mathrm{FC}_{i}+\varepsilon_{2 i} \text {; donde } \mathrm{IE}_{i}^{*}=\mathrm{IE}_{i} \text { si } \mathrm{ID}_{i}^{*} \geq \mu_{i} \text {; es decir, } \mathrm{D}_{I D i}=1 \text {; de lo contrario } \mathrm{IE}_{i}^{*}=0 \text {; } \\
& \mathrm{TI}_{i}^{*}=\delta . \mathrm{IE}^{*}{ }_{i}+\mathrm{X}_{3 i} \cdot \beta_{3}+\varepsilon_{3 i} \text {; donde } \mathrm{D}_{T I i}=1 \mathrm{si} \mathrm{TI}_{i}{ }_{i}>0, \mathrm{D}_{T I i}=0 \text { de lo contrario } \mathrm{D}_{T I i} \text { es cero; } \\
& \mathrm{NTI}_{i}{ }_{i}=\delta . \mathrm{IE}^{*}{ }_{i}+\mathrm{X}_{4 i} \cdot \beta_{4}+\varepsilon_{4 i} \text {; donde } \mathrm{D}_{T I i}=1 \mathrm{si} \mathrm{TI}{ }_{i}>0, \mathrm{D}_{N I i}=0 \text { de lo contrario } \mathrm{D}_{T I i} \text { es cero; } \\
& \ln \operatorname{Prod}_{i}=\varphi_{1} \cdot \mathrm{TI}_{i}^{*}+\varphi_{2} \cdot \mathrm{NTI}_{i}+\varphi_{3} \cdot \mathrm{IE}_{i}^{*}+\mathrm{X}_{5 i} \cdot \beta_{5}+\varepsilon_{5 i}
\end{aligned}
$$


En el cuadro 2 se presenta una lista de las variables dependientes y $X_{j i}$ y las fuentes de datos utilizadas para estimar el conjunto de ecuaciones de este modelo. La mayoría de las variables $X_{j i}$ se tomaron de Crespi y Zúñiga (2010). La ecuación 1 corresponde a la decisión de la empresa $i$ de invertir en actividades de CTI y esta se representa mediante $I D^{*}{ }_{i}$. Específicamente, una empresa decide invertir si $I D^{*}{ }_{i}$ es superior a cero o está en el umbral $\mu_{i}$. Esta variable latente es positiva si las empresas han de hecho invertido en CTI, es decir, si la variable ficticia $D_{I D i}=1 . X_{1 i}$ es el conjunto de factores que afecta el aspecto de apropiabilidad de las decisiones de las empresas de invertir en CTI. Estos son los siguientes: tamaño de la empresa (representado por el número de trabajadores), una variable ficticia para las empresas de exportación $\left(D_{x}\right)$, otra para las empresas extranjeras con una cuota de capital mayor del 10\% $(F O)$ y por último una para la protección de patentes (PatenP, si las empresas tuvieron patentes en el período 2002-2004).

\section{Cuadro 2}

Lista de variables y fuentes de datos del modelo CDM

\begin{tabular}{|c|c|c|}
\hline Nombre & Descripción & Fuente \\
\hline ID & $\begin{array}{l}\text { Variable ficticia con valor uno para las empresas que decidieron } \\
\text { invertir en actividades de CTI, de lo contrario cero. }\end{array}$ & $\begin{array}{l}\text { CONCYTEC/INEI (2005), } \\
\text { sección Vl.1, ítem 42.h }\end{array}$ \\
\hline Dx & $\begin{array}{l}\text { Variable ficticia con valor uno para las empresas que exportaron durante } \\
\text { por lo menos dos años entre } 1993 \text { y } 2004 \text {, de lo contrario cero. }\end{array}$ & SUNAT (2012) \\
\hline F0 & $\begin{array}{l}\text { Variable ficticia con valor uno para las empresas con más del 10\% } \\
\text { del capital total de propiedad extranjera, de lo contrario cero. }\end{array}$ & Peru Top Publications (2000-2007) \\
\hline InSize & Logaritmo natural del número de trabajadores de una empresa. & $\begin{array}{l}\text { CONCYTEC/INEI (2005), } \\
\text { sección I, ítem } 22\end{array}$ \\
\hline PatenP & Variable ficticia con valor uno para las empresas con patentes, de lo contrario cero. & $\begin{array}{l}\text { CONCYTEC/INEI (2005), } \\
\text { sección Vl.1, ítem } 48\end{array}$ \\
\hline PFS & $\begin{array}{l}\text { Variable ficticia con valor uno para las empresas que reciben } \\
\text { apoyo financiero público, de lo contrario cero. }\end{array}$ & $\begin{array}{l}\text { CONCYTEC/INEI (2005), sección } \\
\text { II, ítems 2, } 3 \text { y 4, sección XI, } \\
\text { ítem 42.a; sección II, ítem 1.b }\end{array}$ \\
\hline FC & $\begin{array}{l}\text { Variable ficticia con valor uno para las empresas que declararon que las restricciones } \\
\text { de crédito constituían un obstáculo importante para la innovación, de lo contrario cero. }\end{array}$ & $\begin{array}{l}\text { CONCYTEC/INEI (2005), } \\
\text { sección Vl.1, ítem 46.h }\end{array}$ \\
\hline Dcoord & $\begin{array}{l}\text { Variable ficticia con valor uno para las empresas que se } \\
\text { coordinaron con otras entidades, de lo contrario cero. }\end{array}$ & $\begin{array}{l}\text { CONCYTEC/INEI (2005), } \\
\text { sección VI, ítem } 49\end{array}$ \\
\hline $\mathrm{INFO}_{1}$ & $\begin{array}{l}\text { Variable ficticia con valor uno para las empresas que utilizan Internet para } \\
\text { buscar información sobre productos y procesos, de lo contario cero. }\end{array}$ & $\begin{array}{l}\text { CONCYTEC/INEI (2005), } \\
\text { sección V, ítem } 5.1\end{array}$ \\
\hline $\mathrm{INFO}_{2}$ & $\begin{array}{l}\text { Variable ficticia con valor uno para las empresas que utilizan Internet } \\
\text { para actividades de investigación, de lo contrario cero. }\end{array}$ & $\begin{array}{l}\text { CONCYTEC/INEI (2005), } \\
\text { sección V, ítem } 5.1\end{array}$ \\
\hline $\mathrm{INFO}_{3}$ & $\begin{array}{l}\text { Variable ficticia con valor uno para las empresas que utilizan Internet para buscar } \\
\text { información sobre instituciones gubernamentales, de lo contrario cero. }\end{array}$ & $\begin{array}{l}\text { CONCYTEC/INEI (2005), } \\
\text { sección V, ítem } 5.1\end{array}$ \\
\hline InIE & $\begin{array}{l}\text { Logaritmo natural del valor real de la inversión en CTI de las } \\
\text { empresas con respecto al número de trabajadores. }\end{array}$ & $\begin{array}{l}\text { CONCYTEC/INEI (2005), sección } \\
\text { II, ítems 2, } 3 \text { y 4, sección XI, } \\
\text { ítem 42.a; sección II, ítem 1.b }\end{array}$ \\
\hline $\ln \mid \mathrm{E}^{\mathrm{e}}$ & Valor predicho de InIE a partir de la estimación de Heckman de la ecuación (2). & $\begin{array}{l}\text { CONCYTEC/INEI (2005), sección } \\
\text { II, ítems 2, } 3 \text { y 4, sección XI, } \\
\text { ítem 42.a; sección II, ítem 1.b }\end{array}$ \\
\hline$\underline{D_{\text {control }}}$ & Variable ficticia con valor uno para las empresas con k cero, de lo contrario cero. & \\
\hline $\ln (k+1)$ & $\begin{array}{l}\text { Logaritmo natural del valor real del gasto de capital de } \\
\text { las empresas por trabajador más uno. }\end{array}$ & $\begin{array}{l}\text { CONCYTEC/INEI (2005) } \\
\text { sección II, ítem } 31\end{array}$ \\
\hline InProd & Logaritmo natural del valor real de las empresas por trabajador. & $\begin{array}{l}\text { CONCYTEC/INEI (2005) } \\
\text { sección I, ítems } 22 \text { y 23, }\end{array}$ \\
\hline $\mathrm{Tl}$ & $\begin{array}{l}\text { Variable ficticia con valor uno para las empresas que tienen } \\
\text { innovación tecnológica, de lo contrario cero. }\end{array}$ & $\begin{array}{l}\text { CONCYTEC /INEI (2005), } \\
\text { sección Vl.1, ítem 44.h }\end{array}$ \\
\hline NTI & $\begin{array}{l}\text { Variable ficticia con valor uno para las empresas que tienen } \\
\text { innovación no tecnológica, de lo contrario cero. }\end{array}$ & $\begin{array}{l}\text { CONCYTEC/INEI (2005), } \\
\text { sección Vl.1, ítem 44.h }\end{array}$ \\
\hline
\end{tabular}

Fuente: Elaboración propia, sobre la base de Consejo Nacional de Ciencia, Tecnología e Innovación Tecnológica/Instituto Nacional de Estadística e Informática (CONCYTEC/INEI), Encuesta Nacional de Ciencia, Tecnología e Innovación Tecnológica (ENCYT-04), Lima, 2005; Superintendencia Nacional de Aduanas y Administración Tributaria (SUNAT), Series estadísticas de exportaciones, Lima, 2012; y Peru Top Publications, Peru: The Top 10,000 Companies, Lima, varios años. 
Estas variables son coherentes con varios argumentos considerados en la literatura (por ejemplo, Crepon, Duguet y Mairesse, 1998; Braga y Willmore, 1991; Kumar y Aggarwal, 2005; Álvarez, 2001; Cohen y Klepper, 1996; Benavente, 2006; Crespi y Peirano, 2007; Girma y Görg, 2007). La ecuación (2) es la intensidad de la inversión en CTI de la empresa medida por el gasto en CTI por trabajador. Si la empresa decide invertir, $I E^{*}{ }_{i}$ sería igual al gasto en CTI real por trabajador $I E_{i}$, de lo contrario $I E^{*}{ }_{i}$ sería cero. $X_{2 i}$ es el conjunto de factores que influye en la intensidad de la inversión en CTI de la empresa. Este conjunto será igual a $X_{1 i}$ más las siguientes variables ficticias: apoyo financiero público $(P F S)$, fuentes de información del mercado $\left(I N F O_{1}\right)$, fuentes de información científicas $\left(\mathrm{INFO}_{2}\right)$, información de instituciones gubernamentales (INFO3) y el grado de coordinación, cooperación o colaboración entre la empresa i y otras entidades $\left(D_{\text {coord }}\right)$.

Dichos factores se encuentran en varios estudios sobre América Latina, por ejemplo con respecto a la Argentina y México (Raffo, Lhuillery y Miotti, 2008). Si bien en los argumentos basados en el tamaño (por ejemplo, la explotación de economías de escala y alcance) se indica que el tamaño afecta la ecuación de intensidad de la inversión, la evidencia empírica demuestra que puede afectar las decisiones de inversión pero no la intensidad de la inversión en CTI. Por ese motivo, y para fines de identificación, en este trabajo el tamaño se incluye en la ecuación (1) y se excluye de la ecuación (2). La evidencia peruana que se muestra más adelante sobre los efectos marginales de ambas ecuaciones apoya estos cambios. Por último, el análisis de las limitaciones financieras apoya la inclusión de esta limitación en las ecuaciones (1) y (2).

Las ecuaciones 3 y 4 representan los resultados del proceso de innovación tecnológica $\left(T I^{*} i\right)$ y no tecnológica $\left(N T I^{*} i\right)$ o el rendimiento esperado de la innovación. Las variables latentes $T I^{*}{ }_{i}$ y $N T I^{*}{ }_{i}$ son positivas si las empresas, de hecho, tienen productos de innovación, es decir, si la variable ficticia $D_{T I i}=1 \circ D_{N T I i}=1^{14}$. Ambos productos están determinados por $I E^{*}{ }_{i}$ y el conjunto de factores $X_{3 i}=X_{4 i}$. Las variables de este conjunto incluyen el tamaño y dos variables ficticias correspondientes a las exportaciones y a la propiedad extranjera. Hahn y Park (2010), Hanley y Monreal-Pérez (2011) e Ito (2011) presentan el argumento y la evidencia de una relación entre las exportaciones y la innovación. Crespi y Zúñiga (2010) proporcionan la evidencia de los otros dos factores, propiedad extranjera y tamaño.

La ecuación (5) es la productividad del trabajo de una empresa (medida por el valor agregado real de la empresa por trabajador) determinada por los productos de innovación tecnológica y no tecnológica, la inversión en CTI y $X_{4 i}$, que incluye el tamaño y el capital social por trabajador, $\ln k i$ (en logaritmo natural). Si bien la medición de la productividad tiene diversos defectos, no solo en los productos (Tybout, Katayama y Lu, 2009; Syverson, 2011), sino también en los servicios (Dean y Kunze, 1992; Griliches, 1992; Crepon, Duguet y Mairesse, 1998; Gallouj y Djellal, 2008; Gallouj y Savona, 2009; Crespi y Zúñiga, 2010; Biege y otros, 2011), en este estudio la productividad del trabajo se midió como el valor agregado real (o ventas netas ${ }^{15}$ ) por trabajador $^{16}$.

En las estimaciones, las variables cuantitativas como el tamaño, la productividad y el gasto en inversión se transforman en logaritmos naturales. El resto de las variables (que son binarias) no se transforma. Además, la heterogeneidad en las ramas de los cuatro grupos de la CIIU (Revisión 3)

${ }^{14} \mathrm{NTI}$ es una variable ficticia igual a uno si la empresa muestra resultados o productos de innovación en comercialización y organización, de lo contrario cero.

15 El valor agregado se obtiene mediante el cociente entre el valor agregado y el valor de producción del respectivo sector de la CIIU tomado de los cuadros de insumo-producto de 1994 y 2007 proporcionados en forma preliminar por el INEI.

${ }^{16}$ Los problemas más comunes asociados con las medidas de productividad y producción se relacionan con los deflactores de precios relevantes para calcular valores reales y las medidas de calidad del producto. En el sector de servicios, las medidas de producción se limitarán al monto en valor (ventas o valor agregado) de la transacción (según Griliches, 1992 y Gallouj y Savona, 2009). Las características de la producción de servicios representadas por el denominado criterio IHIP (intangibilidad, I; heterogeneidad, H; inseparabilidad, I; y carácter perecedero, P) y otras consideraciones (por ejemplo en los servicios intensivos en conocimiento) señaladas por Biege y otros (2011) (como el carácter innovador de la producción, la "producción interna", las cifras de insumos y el conocimiento) no se tomarán en cuenta debido a las limitaciones de los datos. 
se introduce mediante una variable binaria $I S I C_{n}$, donde $n$ es el primer dígito de la rama de la CIIU. Para cada grupo de empresas se incluye una rama de la CIIU en la constante para evitar problemas de colinealidad.

Dadas estas transformaciones y variables ficticias, la estrategia econométrica comprende los siguientes pasos: en primer lugar, se estiman las ecuaciones relativas a la decisión de invertir y a la intensidad de la inversión mediante la estimación de un modelo Tobit generalizado (o estimación por máxima verosimilitud de Heckman, asumiendo una distribución conjunta normal para los términos de error de ambas ecuaciones). Para fines de robustez, se aplicó el procedimiento de dos etapas de Heckman o estimador Heckit (que asume una distribución normal condicional para el término de error), que no se detalla debido a limitaciones de espacio. La variable de tamaño incluida en la ecuación $I D^{*}$ y excluida de la ecuación $I E^{*}$ permite la identificación de ambas ecuaciones. Además, $P P S, D_{\text {coord }}$ y el conjunto de variables de información refuerzan la identificación de los parámetros de ambas ecuaciones. Asimismo, para evitar resultados espurios en las ecuaciones (1) y (2), las empresas que no invirtieron en actividades de CTI y respondieron que no tenían ninguna restricción a la producción de innovación se eliminaron de la muestra ${ }^{17}$.

En segundo lugar, los dos productos de la innovación, que corresponden a las ecuaciones (3) y (4), se obtienen mediante una estimación probit (estimación de máxima verosimilitud; cuando se asume que los errores de la ecuación no están correlacionados). Asimismo, ninguna estimación biprobit (cuando se asume que los errores de la ecuación están correlacionados) presentada produjo resultados similares a los de la estimación probit. Para evitar la posible endogeneidad de la intensidad de la inversión en CTI, también se utilizaron los valores predichos a partir de la ecuación de intensidad de la inversión en CTI estimada en lugar de los valores reales de $I E$. En esos casos, los errores estándar se estimaron mediante la técnica de bootstrap.

En tercer lugar, la productividad (ecuación (5)) se obtiene mediante estimaciones de mínimos cuadrados con errores estándar de bootstrap cuando los valores predichos de TI, NTI e IE se utilizaron como variables exógenas. Estas estimaciones no se presentan aunque los resultados no se diferencian de los detallados en el cuadro 7. Por último, para evitar la reducción del tamaño de la muestra correspondiente a cada sector para las empresas sin información sobre $k i$, la variable $\ln k_{i}$ se reemplazó con $\ln \left(1+k_{i}\right)$ más una variable ficticia de control $\left(D_{\text {control }}\right.$ igual a uno cuando $k_{i}=0$, de lo contrario cero). Además, se evitaron los problemas de colinealidad al no incluir TI, NTI e IE (valor real o predicho) en la estimación de la ecuación de la productividad del trabajo ${ }^{18}$.

\section{Estimación y resultados}

En los cuadros 3 a 7 se muestran los coeficientes de regresión y las estadísticas de los métodos de estimación implementados para el conjunto de ecuaciones del modelo utilizando la muestra descrita en la sección de descripción de los datos ${ }^{19}$. Las estimaciones de la ecuación (1) indican que solo el tamaño (en su versión no censurada) parece afectar la decisión de las empresas de

\footnotetext{
${ }^{17}$ El autor desea agradecer a Gustavo Crespi por sus comentarios con respecto a la reducción de la muestra. Con esa eliminación, la muestra en las ecuaciones selectiva y de intensidad de la inversión se redujo a 2.896 empresas.

18 Cabe señalar que en el grupo de los servicios intensivos en conocimiento de la CIIU, el 10,2\% de las empresas produjo solamente innovación tecnológica, el 13\% produjo solo innovación no tecnológica y el 18\% produjo ambas. En los servicios tradicionales, las respectivas proporciones fueron el 6\%, el 16\% y el 17\%. En las empresas manufactureras de alta tecnología, las respectivas proporciones fueron el $15 \%$, el $7 \%$ y el $35 \%$ y en las empresas manufactureras de baja tecnología el 16\%, el $7 \%$ y el $24 \%$.

19 La muestra presenta un sesgo hacia las empresas medianas y grandes con una media de 68 trabajadores cada una para el modelo básico y 79 para el modelo ampliado. Debido a que, en general, estas empresas pueden tener una mayor probabilidad de invertir en actividades de CTI, los coeficientes estimados podrían estar sobreestimados con respecto a los coeficientes provenientes de una muestra con empresas más grandes.
} 
invertir en actividades de CTI en todas las ramas de actividad, aunque los efectos marginales o censurados para las empresas que decidieron invertir no fueron estadísticamente significativos. Por otra parte, las restricciones financieras ${ }^{20}$ parecen haber limitado las decisiones de inversión solo en los servicios tradicionales. No obstante, una vez que las empresas decidieron invertir, el efecto de estas restricciones no fue significativo desde el punto de vista estadístico ${ }^{21}$. Los coeficientes del resto de los factores no fueron estadísticamente significativos.

Cuadro 3

Coeficientes censurados (C) y no censurados (NC) de la ecuación selectiva (1) (observada), relativa a la decisión de las empresas de invertir en ciencia, tecnología e innovación (CTI), por tipo de empresa: Tobit generalizado (selección de Heckman)

\begin{tabular}{|c|c|c|c|c|c|c|c|c|}
\hline \multirow{2}{*}{$\begin{array}{l}\text { Sectores } \\
\text { Var. } \\
\end{array}$} & \multicolumn{2}{|c|}{$\begin{array}{l}\text { Servicios intensivos } \\
\text { en conocimiento }\end{array}$} & \multicolumn{2}{|c|}{ Servicios tradicionales } & \multicolumn{2}{|c|}{$\begin{array}{l}\text { Manufacturero de } \\
\text { alta tecnología }\end{array}$} & \multicolumn{2}{|c|}{$\begin{array}{l}\text { Manufacturero de } \\
\text { baja tecnología }\end{array}$} \\
\hline & C & $\mathrm{NC}$ & C & NC & C & $\mathrm{NC}$ & C & NC \\
\hline $\mathrm{Dx}$ & 0,033 & 0,083 & -0001 & $-0,001$ & $-0,037$ & $-0,318$ & 0,022 & 0,065 \\
\hline FO & $-0,046$ & $-0,116$ & $-0,1054$ & $-0,136$ & $-0,079$ & $-0,508$ & 0,057 & 0,170 \\
\hline$|S| C_{1}$ & & & & & & & $-0,008$ & $-0,023$ \\
\hline $\mathrm{ISIC}_{2}$ & & & & & $-0,005$ & $-0,041$ & $-0,028$ & $-0,080$ \\
\hline $\mathrm{ISIC}_{3}$ & & & $-0,460$ & $-5,832$ & & & & \\
\hline $\mathrm{ISIC}_{4}$ & & & $-0,058$ & $-0,147$ & & & & \\
\hline $\mathrm{ISIC}_{5}$ & & & $-0,012$ & $-0,031$ & & & & \\
\hline $\mathrm{ISIC}_{7}$ & $-0,046$ & $-0,116$ & $-0,128$ & $-0,332^{\star \star}$ & & & & \\
\hline $\mathrm{ISIC}_{8}$ & & & 0,170 & $0,430^{\star}$ & & & & \\
\hline $\mathrm{FC}$ & $-0,077$ & $-0,193$ & $-0,125$ & $-0,321^{\star \star \star}$ & $-0,024$ & $-0,192$ & $-0,025$ & $-0,070$ \\
\hline PFS & 0,563 & 6,158 & 0,601 & 5,406 & 0,333 & 6,522 & 0,488 & 6,377 \\
\hline PatenP & 0,483 & 5,468 & 0,588 & 6,413 & 0,143 & 6,354 & 0,395 & 6,484 \\
\hline InSize & 0,112 & $0,282^{\star \star \star}$ & 0,103 & $0,260^{\star * *}$ & 0,071 & $0,607^{\star \star \star}$ & 0,072 & $0,208^{* \star \star}$ \\
\hline Const. & & $-0,99^{\star \star \star}$ & & $-0,914^{\star \star \star}$ & & $-1,67^{\star \star \star}$ & & $-0,86^{\star}$ \\
\hline Observ. & 539 & 539 & 1411 & 1411 & 178 & 178 & 768 & 768 \\
\hline$\rho$ & & $0,610^{\star \star}$ & & $0,873^{\star \star \star}$ & & 0,221 & & 0,158 \\
\hline$\sigma$ & & 2,171 & & 2,512 & & 1,630 & & 1,960 \\
\hline$\lambda$ & & 1,326 & & 2,193 & & 0,361 & & 0,309 \\
\hline V. pred. & 0,541 & & 0,453 & & 0,941 & & 0,701 & \\
\hline V. obs. & 0,445 & & 0,393 & & 0,567 & & 0,465 & \\
\hline
\end{tabular}

Fuente: Elaboración propia.

Nota: * nivel de significación del 10\%; ** nivel de significación del 5\%; ${ }^{\star \star \star}$ nivel de significación de menos del 1\%.

20 La proporción de empresas con restricciones financieras (que las describieron como un obstáculo importante a la innovación) con respecto al total de empresas en cada grupo de la CIIU fue del $21,2 \%$ en los servicios intensivos en conocimiento, el $18,5 \%$ en los servicios tradicionales, el 34,3\% en las empresas manufactureras de alta tecnología, el 29,4\% en las empresas manufactureras de baja tecnología y el 22,9\% en los servicios y las manufacturas en general.

${ }^{21}$ Los resultados de la estimación realizada con el procedimiento en dos etapas de Heckman (es decir Heckit) que no se presentan aquí fueron mucho mejores para la ecuación relativa a la decisión de invertir. Así, los coeficientes censurados y no censurados del tamaño de la empresa fueron estadísticamente significativos para todos los sectores. Por otra parte, los coeficientes censurados o marginales del apoyo financiero público y la protección de patentes fueron estadísticamente significativos para todos los sectores. Las variables ficticias relativas al carácter exportador y a la propiedad extranjera resultaron no significativas o de dudosa significación estadística. Por último, el coeficiente de restricciones financieras también fue estadísticamente significativo para los servicios tradicionales. 
Los resultados econométricos con respecto a la intensidad de la inversión en CTI (ecuación (2)) son más variados. El carácter de empresa exportadora, el apoyo financiero público, las restricciones financieras y la coordinación con otras entidades fueron factores estadísticamente importantes en el monto de la inversión en CTI de las empresas en las ramas de los servicios tradicionales. Los primeros dos factores contribuyeron a aumentar la intensidad de la inversión y los dos últimos a reducirla. Sin embargo, una vez que una empresa decidió invertir, solo el coeficiente marginal de coordinación fue estadísticamente significativo y contribuyó a reducir el monto de la inversión en actividades de CTI. En el caso de las ramas de servicios intensivos en conocimiento y manufacturera de baja tecnología, el apoyo financiero público fue el factor más importante y significativo desde el punto de vista estadístico. Una vez más, los respectivos coeficientes marginales no fueron significativos.

Además, en el caso de los servicios intensivos en conocimiento, los respectivos coeficientes censurados y no censurados fueron estadísticamente significativos para las empresas que utilizaban Internet para buscar información sobre productos y procesos ( $\left(\mathrm{NFO}_{1}\right)$, para actividades de investigación $\left(\mathrm{INFO}_{2}\right)$ y para obtener información sobre instituciones gubernamentales $\left(\mathrm{INFO}_{3}\right)$. Las empresas que utilizaban Internet para los primeros dos usos presentaban un mayor nivel de intensidad de la inversión, mientras que aquellas que la utilizaban para el tercer uso invirtieron menos en actividades de CTI.

\section{Cuadro 4}

Coeficientes censurados (C) y no censurados (NC) de la ecuación (2) (observada), relativa a la intensidad de la inversión en ciencia, tecnología e innovación (CTI), por tipo de empresa: Tobit generalizado (selección de Heckman)

\begin{tabular}{|c|c|c|c|c|c|c|c|c|}
\hline \multirow{2}{*}{$\begin{array}{l}\text { Sectores } \\
\text { Var. } \\
\end{array}$} & \multicolumn{2}{|c|}{$\begin{array}{l}\text { Servicios intensivos } \\
\text { en conocimiento }\end{array}$} & \multicolumn{2}{|c|}{ Servicios tradicionales } & \multicolumn{2}{|c|}{$\begin{array}{l}\text { Manufacturero de } \\
\text { alta tecnología }\end{array}$} & \multicolumn{2}{|c|}{$\begin{array}{c}\text { Manufacturero de } \\
\text { baja tecnología }\end{array}$} \\
\hline & C & NC & C & NC & C & NC & C & NC \\
\hline Dx & 0,555 & 0,621 & 0,670 & $0,668^{* *}$ & 0,100 & 0,076 & $-0,121$ & $-0,111$ \\
\hline FO & 0,232 & 0,136 & 0,435 & 0,233 & $-0,615$ & $-0,663$ & 0,375 & 0,401 \\
\hline PFS & 1,748 & $2,943^{\star \star \star}$ & 0,676 & $2,796^{\star \star \star}$ & 0,447 & 0,644 & 0,486 & $0,727^{\star}$ \\
\hline Dcoord & $-0,560$ & $-0,560$ & $-0,475^{\star \star}$ & $-0,475^{\star *}$ & 0,450 & 0,450 & 0,313 & 0,313 \\
\hline $\mathrm{INFO}_{1}$ & $1,136^{\star \star}$ & $1,136^{\star \star}$ & 0,178 & 0,178 & $-0,612$ & $-0,612$ & 0,443 & 0,443 \\
\hline $\mathrm{INFO}_{2}$ & $0,723^{\star \star}$ & $0,723^{\star \star}$ & 0,278 & 0,278 & $0,779^{*}$ & $0,779^{*}$ & $-0,055$ & $-0,055$ \\
\hline $\mathrm{INFO}_{3}$ & $-1,066^{\star *}$ & $-1,066^{\star \star}$ & 0,240 & 0,240 & $-0,098$ & $-0,098$ & $-0,441$ & $-0,441$ \\
\hline $\mathrm{ISIC}_{1}$ & & & & & & & $-0,244$ & $-0,247$ \\
\hline $\mathrm{ISIC}_{2}$ & & & & & $-0,036$ & $-0,039$ & 0,128 & 0,116 \\
\hline $\mathrm{ISIC}_{3}$ & & & 13,969 & 2,501 & & & & \\
\hline $\mathrm{ISIC}_{4}$ & & & 0,550 & 0,332 & & & & \\
\hline $\mathrm{ISIC}_{5}$ & & & 0,047 & 0,002 & & & & \\
\hline $\mathrm{ISIC}_{7}$ & $-0,115$ & $-0,210$ & $-0,121$ & $-0,621$ & & & & \\
\hline $\mathrm{ISIC}_{8}$ & & & $-0,207$ & 0,374 & & & & \\
\hline FC & $-0,372$ & $-0,532$ & $-0,243$ & $-0,722^{\star *}$ & 0,129 & 0,114 & $-0,244$ & $-0,255$ \\
\hline PatenPa & 0,540 & 1,561 & $-2,070$ & & 0,587 & 0,682 & $-0,197$ & \\
\hline InSize & $-0,229$ & & $-0,378$ & & $-0,046$ & & $-0,033$ & \\
\hline Const. & & $4,516^{\star \star \star}$ & & $3,381^{\star \star \star}$ & & $6,090^{\star \star *}$ & & $5,910^{\star \star \star}$ \\
\hline Obser. & 539 & 539 & 1411 & 1411 & 178 & 178 & 768 & 768 \\
\hline V. pred. & 5,786 & & 5,637 & & 6,062 & & 5,978 & \\
\hline
\end{tabular}

Fuente: Elaboración propia.

Nota: * nivel de significación del 10\%; ** nivel de significación del 5\%; ${ }^{\star \star \star}$ nivel de significación de menos del 1\%.

a La variable de protección de patentes (PatenP) se excluyó de los servicios tradicionales y las empresas manufactureras de baja tecnología debido a problemas de concavidad en la función de verosimilitud logarítmica. 
Por último, en el caso de las empresas manufactureras de alta tecnología, solo los coeficientes censurados y no censurados del uso de Internet para actividades de investigación fueron estadísticamente significativos para la inversión en actividades de CTI.

Las empresas que utilizaron ese tipo de información registraron montos de inversión superiores $^{22}$. En el caso de las estimaciones de innovación, las ecuaciones (3) y (4), los resultados fueron más uniformes en todas las ramas de actividad con respecto a los de la ecuación (2).

Cuadro 5

Coeficientes marginales de la ecuación (3) (observada), relativa a la producción de innovación tecnológica, por tipo de empresa: método probit

\begin{tabular}{|c|c|c|c|c|c|c|c|c|}
\hline \multirow[b]{2}{*}{ InIE } & \multicolumn{2}{|c|}{$\begin{array}{l}\text { Servicios intensivos } \\
\text { en conocimiento }\end{array}$} & \multicolumn{2}{|c|}{$\begin{array}{l}\text { Servicios } \\
\text { tradicionales }\end{array}$} & \multicolumn{2}{|c|}{$\begin{array}{l}\text { Manufacturero de } \\
\text { alta tecnología }\end{array}$} & \multicolumn{2}{|c|}{$\begin{array}{l}\text { Manufacturero de } \\
\text { baja tecnología }\end{array}$} \\
\hline & $0,108^{\star * *}$ & & $0,062^{\star \star *}$ & & $0,169^{\star \star *}$ & & $0,146^{\star \star *}$ & \\
\hline $\ln \mathrm{E}^{\mathrm{a}}$ & & $0,139^{\star \star \star}$ & & $0,125^{\star \star \star}$ & & $0,348^{\star *}$ & & $0,368^{* * *}$ \\
\hline InSize & $0,055^{\star \star \star}$ & $0,087^{\star \star \star}$ & $0,039^{\star \star \star}$ & $0,041^{\star \star \star}$ & $0,175^{\star \star \star}$ & $0,166^{\star \star \star}$ & $0,068^{\star \star \star}$ & $0,064^{\star \star \star}$ \\
\hline Dx & 0,022 & $-0,044$ & 0,025 & $-0,028$ & $-0,091$ & $-0,051$ & 0,062 & $0,100^{\star \star *}$ \\
\hline FO & 0,041 & $-0,040$ & 0,014 & 0,008 & $-0,167$ & $-0,017$ & 0,028 & $-0,115^{*}$ \\
\hline $\mathrm{ISIC}_{1}$ & & & & & & & 0,028 & 0,081 \\
\hline $\mathrm{ISIC}_{2}$ & & & & & 0,073 & 0,008 & 0,027 & $-0,043$ \\
\hline $\mathrm{ISIC}_{3}$ & & & 0,059 & $-0,170^{\star \star \star}$ & & & & \\
\hline $\mathrm{ISIC}_{4}$ & & & $-0,019$ & $-0,026$ & & & & \\
\hline $\mathrm{ISIC}_{5}$ & & & $-0,052$ & $-0,092^{\star \star}$ & & & & \\
\hline $\mathrm{ISIC}_{6}$ & & & & $-0,343^{\star \star \star}$ & & & & \\
\hline $\mathrm{ISIC}_{7}$ & 0,049 & 0,068 & $-0,033$ & $-0,006$ & & & & \\
\hline $\mathrm{ISIC}_{8}$ & & & $0,193^{*}$ & 0,131 & & & & \\
\hline Obser. & 539 & 586 & 1411 & 1534 & 178 & 200 & 768 & 874 \\
\hline Prob. pred. & 0,172 & 0,334 & 0,144 & 0,340 & 0,397 & 0,412 & 0,279 & 0,305 \\
\hline
\end{tabular}

Fuente: Elaboración propia.

Nota: $\quad$ * nivel de significación del 10\%; ${ }^{* \star}$ nivel de significación del 5\%; ${ }^{* \star}$ nivel de significación de menos del 1\%.

a Predicho con el método de Heckman y con errores estándar de bootstrap para la variable independiente.

\footnotetext{
22 Las estimaciones realizadas con el método Heckit que no se presentan aquí produjeron coeficientes más robustos para el apoyo financiero público. Otros factores también fueron más importantes según la estimación Heckit. Así, los coeficientes de restricciones financieras fueron estadísticamente robustos para las dos ramas de servicios de la CIIU (y para los sectores manufacturero y de servicios en general). En forma análoga, el efecto de la variable de protección de patentes en la intensidad de la inversión en CTI fue estadísticamente significativo para los servicios tradicionales, los servicios en general, el sector manufacturero de baja tecnología, el sector manufacturero en general y para todos los sectores (manufacturero y de servicios en general). Por último, el carácter de empresa exportadora afectó la intensidad de la inversión en CTI de manera positiva. El resto de los factores no fueron robustos o carecieron de significación estadística.
} 


\section{Cuadro 6}

Coeficientes marginales de la ecuación (4) (observada), relativa a la producción de innovación no tecnológica, por tipo de empresa: método probit

\begin{tabular}{|c|c|c|c|c|c|c|c|c|}
\hline \multirow[b]{2}{*}{$\ln \mathrm{IE}$} & \multicolumn{2}{|c|}{$\begin{array}{l}\text { Servicios intensivos } \\
\text { en conocimiento }\end{array}$} & \multicolumn{2}{|c|}{$\begin{array}{l}\text { Servicios } \\
\text { tradicionales }\end{array}$} & \multicolumn{2}{|c|}{$\begin{array}{l}\text { Manufacturero de } \\
\text { alta tecnología }\end{array}$} & \multicolumn{2}{|c|}{$\begin{array}{c}\text { Manufacturero de } \\
\text { baja tecnología }\end{array}$} \\
\hline & $0,097^{\star \star \star}$ & & $0,133^{\star \star \star}$ & & $0,169^{\star \star \star}$ & & $0,082^{\star \star \star}$ & \\
\hline $\operatorname{In} \mathrm{E}^{\mathrm{a}}$ & & $0,064^{\star \star *}$ & & $0,151^{\star \star *}$ & & $0,128^{\star \star}$ & & 0,081 \\
\hline InSize & $0,030^{\star \star}$ & $0,065^{\star \star \star}$ & $0,062^{\star \star \star}$ & $0,054^{\star \star \star}$ & $0,175^{\star \star \star}$ & $0,114^{\star \star \star *}$ & $0,034^{\star \star \star}$ & $0,060^{\star \star \star}$ \\
\hline $\mathrm{Dx}$ & $-0,094^{*}$ & $-0,086$ & $-0,095^{\star \star \star}$ & $-0,101^{\star \star \star}$ & $-0,091$ & $-0,082$ & $-0,046$ & $-0,014$ \\
\hline FO & $-0,119^{\star \star}$ & $-0,118^{*}$ & $-0,166^{\star \star \star}$ & $-0,110^{\star \star \star}$ & $-0,167$ & 0,020 & 0,070 & 0,103 \\
\hline $\mathrm{ISIC}_{1}$ & & & & & & & $-0,061$ & $-0,048$ \\
\hline $\mathrm{ISIC}_{2}$ & & & & & 0,073 & $-0,048$ & $-0,115$ & $-0,103$ \\
\hline $\mathrm{ISIC}_{3}$ & & & & $-0,313^{\star \star \star}$ & & & & \\
\hline $\mathrm{ISIC}_{4}$ & & & $-0,012$ & $-0,083$ & & & & \\
\hline $\mathrm{ISIC}_{5}$ & & & $0,0896^{\star}$ & 0,022 & & & & \\
\hline $\mathrm{ISIC}_{6}$ & & & & $-0,343^{\star \star *}$ & & & & \\
\hline $\mid \mathrm{SIC}_{7}$ & $-0,0635$ & $-0,048$ & 0,011 & 0,010 & & & & \\
\hline $\mathrm{ISIC}_{8}$ & & & 0,044 & 0,019 & & & & \\
\hline Obser. & 539 & 586 & 1407 & 1534 & 178 & 200 & 768 & 874 \\
\hline Prob. pred. & 0,230 & 0,334 & 0,199 & 0,340 & 0,397 & 0,412 & 0,200 & 0,305 \\
\hline
\end{tabular}

Fuente: Elaboración propia.

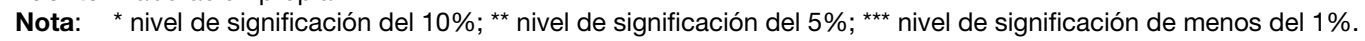

a Predicho con el método de Heckman y con errores estándar de bootstrap para la variable independiente.

Los coeficientes de intensidad de la inversión y tamaño fueron positivos y estadísticamente significativos para los cuatro grupos de ramas de actividad y los dos tipos de innovación ${ }^{23}$. Además, entre las empresas manufactureras de baja tecnología, las empresas exportadoras presentaron una mayor probabilidad de producir innovación tecnológica que las empresas orientadas al mercado interno. Por el contrario, las empresas extranjeras son menos propensas a producir innovación tecnológica que las empresas nacionales dentro de esa categoría del sector manufacturero. En el caso de los servicios intensivos en conocimiento y los servicios tradicionales, las empresas orientadas al mercado interno y nacionales parecen tener una mayor probabilidad de producir innovación no tecnológica que las empresas exportadoras y extranjeras ${ }^{24}$.

Por último, las estimaciones de la última ecuación (5) produjeron un resultado robusto para casi todos los grupos de la CIIU. La relación entre capital y trabajo fue el factor más importante y significativo estadísticamente para la productividad del trabajo de las empresas. La intensidad de la inversión también fue más importante para los grupos de la CIIU que para las empresas manufactureras de alta tecnología ${ }^{25}$.

\footnotetext{
${ }^{23}$ Se obtuvieron prácticamente los mismos resultados con los valores predichos de InIE utilizando el método Heckit.

${ }^{24}$ Los servicios tradicionales incluyen a los comerciantes exportadores de bienes primarios de exportación. Cuando los valores predichos de InIE se estimaron con el método Heckit, los resultados del tamaño y la intensidad de la inversión para las ecuaciones NTI y TI fueron similares. Sin embargo, en el caso de la ecuación NTI, el efecto negativo de los exportadores no fue robusto estadísticamente y la propiedad extranjera también afectó a las empresas del sector de servicios intensivos en conocimiento.

25 Los efectos de TI y NTI para todas las ramas de servicios de la CIIU y el sector manufacturero de baja tecnología fueron estadísticamente robustos y positivos cuando los valores predichos de las variables TI se estimaron con el método Heckit para el modelo ampliado. La significación estadística del resto de los factores es similar a los resultados hallados utilizando el método de Heckman.
} 
Cuadro 7

Coeficientes de regresión de la ecuación (5), relativa a la productividad del trabajo, con errores estándar de bootstrap para los valores predichos mediante la estimación de Heckman: modelo ampliado

\begin{tabular}{|c|c|c|c|c|c|c|c|c|}
\hline \multirow[b]{2}{*}{ InSize } & \multicolumn{4}{|c|}{ Servicios intensivos en conocimiento } & \multicolumn{4}{|c|}{ Servicios tradicionales } \\
\hline & $-0,022$ & $-0,025$ & $-0,008$ & $-0,037$ & $-0,183^{\star \star *}$ & $-0,180^{\star \star \star}$ & $-0,183^{\star \star \star}$ & $-0,190^{\star * *}$ \\
\hline $\ln (k+1)$ & $0,138^{\star * *}$ & $0,138^{\star \star \star}$ & $0,139^{\star \star \star}$ & $0,119^{\star \star *}$ & $0,231^{\star \star *}$ & $0,229^{\star * *}$ & $0,230^{\star \star *}$ & $0,236^{\star * *}$ \\
\hline Dcontrol & $0,646^{\star *}$ & $0,639^{\star \star}$ & $0,643^{\star \star}$ & 0,496 & $1,374^{\star \star \star}$ & $1,359^{\star \star \star}$ & $1,373^{\star \star \star}$ & $1,475^{\star \star *}$ \\
\hline $\mathrm{TI}$ & $0,214^{*}$ & 0,153 & & & $-0,006$ & 0,033 & & \\
\hline NTI & $-0,142$ & & $-0,056$ & & 0,081 & & 0,079 & \\
\hline InIE & & & & $0,034^{\star *}$ & & & & $0,039^{\star \star \star}$ \\
\hline $\mathrm{ISIC}_{3}$ & & & & & 0,568 & 0,558 & 0,567 & 0,345 \\
\hline $\mathrm{ISIC}_{4}$ & & & & & $1,217^{\star \star \star}$ & $1,218^{\star \star \star}$ & $1,217^{\star \star \star}$ & $1,137^{\star \star \star}$ \\
\hline $\mathrm{ISIC}_{5}$ & & & & & $1,445^{\star \star \star}$ & $1,450^{\star \star \star}$ & $1,445^{\star \star \star}$ & $1,453^{\star \star \star}$ \\
\hline $\mathrm{ISIC}_{7}$ & $-0,282^{\star \star \star}$ & $-0,273^{\star \star \star}$ & $-0,264^{\star \star}$ & $-0,207^{*}$ & 0,168 & 0,165 & 0,169 & 0,186 \\
\hline $\mathrm{ISIC}_{8}$ & & & & & $-0,454^{*}$ & $-0,453^{*}$ & $-0,455^{\star}$ & $-0,477^{\star}$ \\
\hline Const. & $9,247^{\star \star \star}$ & $9,231^{\star \star \star}$ & $9,239^{\star \star \star}$ & $9,374^{\star \star \star}$ & $8,550^{\star \star \star}$ & $8,571^{* \star *}$ & $8,550^{\star \star \star}$ & $8,407^{\star \star \star}$ \\
\hline Obser. & 570 & 570 & 570 & 524 & 1474 & 1474 & 1474 & 1357 \\
\hline Adj- $R^{2}$ & 0,0455 & 0,0447 & 0,0420 & 0,0440 & 0,234 & 0,234 & 0,234 & 0,240 \\
\hline \multirow[t]{2}{*}{$R^{2}$} & 0,0556 & 0,0531 & 0,0504 & 0,0532 & 0,239 & 0,238 & 0,239 & 0,245 \\
\hline & \multicolumn{4}{|c|}{ Manufacturero de alta tecnología } & \multicolumn{4}{|c|}{ Manufacturero de baja tecnología } \\
\hline InSize & 0,033 & 0,031 & 0,034 & 0,012 & $-0,001$ & 0,002 & 0,001 & $-0,011$ \\
\hline $\ln (k+1)$ & $0,104^{*}$ & $0,103^{*}$ & $0,103^{*}$ & 0,087 & $0,187^{\star \star *}$ & $0,184^{\star * *}$ & $0,187^{\star * \star}$ & $0,198^{\star * *}$ \\
\hline$\underline{D_{\text {control }}}$ & $0,758^{*}$ & $0,753^{*}$ & $0,755^{\star}$ & 0,648 & $1,062^{\star \star \star}$ & $1,043^{\star \star \star}$ & $1,059^{\star \star \star}$ & $1,170^{\star \star \star}$ \\
\hline $\mathrm{TI}$ & 0,006 & $-0,027$ & & & 0,053 & 0,094 & & \\
\hline NTI & $-0,062$ & & $-0,059$ & & 0,092 & & 0,119 & \\
\hline $\ln \mid \mathrm{E}$ & & & & 0,002 & & & & $0,039^{\star \star \star}$ \\
\hline $\mid S I C_{1}$ & & & & & $-0,163$ & $-0,169$ & $-0,160$ & $-0,260$ \\
\hline $\mathrm{ISIC}_{2}$ & 0,123 & 0,125 & 0,123 & 0,177 & 0,153 & 0,143 & 0,157 & 0,049 \\
\hline Const. & $8,862^{\star \star \star}$ & $8,860^{\star \star \star}$ & $8,864^{\star \star \star}$ & $8,996^{\star \star \star}$ & $8,253^{\star \star \star}$ & $8,283^{\star \star \star}$ & $8,259^{\star \star \star}$ & $8,244^{\star * *}$ \\
\hline Obser. & 189 & 189 & 189 & 167 & 836 & 836 & 836 & 735 \\
\hline $\mathrm{Adj}-R^{2}$ & $-0,00886$ & $-0,00410$ & $-0,00336$ & $-0,0124$ & 0,0978 & 0,0977 & 0,0984 & 0,114 \\
\hline$R^{2}$ & 0,0233 & 0,0226 & 0,0233 & 0,0181 & 0,105 & 0,104 & 0,105 & 0,121 \\
\hline
\end{tabular}

Fuente: Elaboración propia.

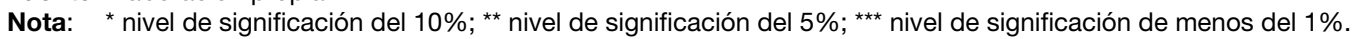

\section{Conclusiones}

En la última década, los responsables institucionales de la formulación de políticas no han dado mucha prioridad al área de ciencia, tecnología e innovación tecnológica en la economía peruana. El Sistema Nacional de Ciencia y Tecnología e Innovación Tecnológica (SINACYT) no se ha articulado institucionalmente y se ha concentrado en fondos y programas particulares dirigidos a promover las actividades de innovación de las empresas en los sectores primario y manufacturero sin una estrategia de innovación específica diseñada previamente. La CTI en los servicios se orientó principalmente a brindar infraestructura de TIC conforme a los principios de acceso universal, asequibilidad, promoción de la competencia privada y convergencia tecnológica de acuerdo con la evolución y el desarrollo de las TIC. 
Sobre la base de datos de una encuesta de empresas sobre CTI realizada en 2004 (CONCYTEC/ INEI, 2005a), se presentó evidencia sólida de los efectos positivos de las actividades de ciencia, tecnología e innovación de las empresas en la productividad del trabajo en los sectores de servicios y manufactureros del Perú. Asignar mayor prioridad a la política de CTI y su efectiva implementación en el país podría mejorar el desempeño mediocre de la década pasada y favorecer el crecimiento de la productividad (Tello, 2012b). Se estimaron dos modelos CDM con métodos que permiten superar los problemas de selección y endogeneidad.

En general, los resultados estadísticos apoyan parcialmente algunas de las hipótesis encontradas en la literatura. Específicamente, el tamaño de las empresas parece ser un determinante clave en la decisión de invertir en actividades de CTI en los siete grupos de la CIIU considerados en el análisis. Sin embargo, para aquellas empresas motivadas para invertir, la protección de patentes - en particular en el caso de las empresas manufactureras - fue un factor determinante para una inversión efectiva. Al mismo tiempo, las restricciones financieras influyeron en las decisiones de las empresas de invertir o no en actividades de CTI solo en el grupo de la CIIU correspondiente a los servicios tradicionales. Se observa que la significación estadística de las restricciones financieras desapareció en el caso de las empresas que decidieron invertir en actividades de CTI, de manera que el factor financiero constituía una limitación relevante solo para las empresas que en última instancia no invirtieron.

En segundo lugar, aunque el apoyo financiero público parece incrementar la variable latente de la intensidad de la inversión de las empresas en la mayoría de los grupos de la CIIU (con excepción de las empresas manufactureras de alta tecnología), el efecto en la intensidad de la inversión real (medida a través de los gastos en actividades de CTI por trabajador) no fue estadísticamente significativo para las empresas que invirtieron en actividades de CTI. Esto significa que las políticas de apoyo público parecen tener un efecto más inductivo (que consiste en hacer que las empresas que no gastan lo hagan) que intensificador (que consiste en aumentar la intensidad de las empresas que ya gastan). El mismo resultado se obtiene con respecto a las restricciones financieras en el grupo de la CIIU correspondiente a los servicios tradicionales. El efecto de otros factores en la intensidad de la inversión en CTI de las empresas también fue estadísticamente robusto para algunos grupos de la CIIU. Específicamente, la información de Internet sobre productos y procesos fue significativa para los servicios intensivos en conocimiento, los servicios en general y para todas las empresas de la muestra (manufactureras y de servicios), mientras que la información de Internet sobre actividades de investigación fue significativa para los servicios intensivos en conocimiento, las empresas manufactureras de alta tecnología, los servicios tradicionales y ambos sectores (servicios y manufacturas) en general, y la coordinación de las empresas con otras entidades para fines de innovación lo fue para los servicios tradicionales y los servicios totales.

En tercer lugar, el tamaño de la empresa y la intensidad de la inversión fueron los determinantes clave para la producción de innovación tecnológica y no tecnológica en todos los grupos de la CIIU. En algunos de ellos (como los servicios y el sector manufacturero de baja tecnología), las empresas orientadas al mercado doméstico y nacionales eran más propensas a elaborar productos de innovación tecnológica y no tecnológica que las empresas exportadoras y de propiedad extranjera. Este resultado puede indicar una mayor necesidad de realizar cambios tecnológicos para competir con las empresas exportadoras y extranjeras en el primer grupo de empresas. Por último, el capital por trabajador y la intensidad de la inversión en CTI afectaron la productividad del trabajo de las empresas en forma positiva para la mayoría de los grupos de la CllU (con excepción del sector manufacturero de alta tecnología).

Desde la perspectiva de la política económica, estos resultados sugieren que las políticas horizontales de CTI (por lo menos para los sectores de servicios y manufactureros) que alientan a las empresas a incrementar la intensidad de sus inversiones en CTI pueden contribuir a aumentar la productividad del trabajo de las empresas. Sin embargo, dado que los productos de innovación no 
mostraron ningún efecto estadístico en la productividad, es necesario realizar un análisis detallado a nivel micro de lo que las empresas consideran productos de innovación y obtener información acerca de aquellos que pueden aumentar la productividad del trabajo. Por último, el hecho de que las actividades de CTI de la mayoría de las empresas se lleven a cabo de manera aislada -la producción de innovación de cualquier tipo, ya sea de productos, procesos, comercialización u organización, se realiza con los fondos propios de las empresas y sin colaboración con otras entidades - y la falta de significación estadística del efecto de la coordinación de las empresas con otras entidades en la intensidad de la inversión en CTI indican la necesidad de explotar las interacciones de las empresas con otras empresas, instituciones de investigación y el gobierno para aumentar la probabilidad de producir innovación ${ }^{26}$ y reducir el gasto en CTI de las empresas por trabajador.

\section{Bibliografía}

Álvarez, R. (2001), "External sources of technological innovation in Chilean manufacturing industry", Estudios de Economía, vol. 28, № 1, Santiago, Universidad de Chile.

Álvarez, R. y G. Crespi (2011), "Financing Gaps, Innovation Gaps? New Evidence from Chile", inédito.

Balboni, M., S. Rovira y S. Vergara (eds.) (2011), ICT in Latin America: A Micro-Data Analysis (LC/R.2172), Santiago, Comisión Económica para América Latina y el Caribe (CEPAL).

Banco Mundial (2013), World Development Indicators [en línea] http://data.worldbank.org/data-catalog/ world-development-indicators.

Benavente M.J. (2006), "The role of research and innovation in promoting productivity in Chile", Economics of Innovation and New Technology, vol. No 4-5, Taylor \& Francis.

Biege, S. y otros (2011), "Challenges of measuring service productivity in innovative, knowledge-intensive business services", Karlsruhe, Fraunhofer Institute for Systems and Innovation Research (ISI).

Braga, H. y L. Willmore (1991), "Technological imports and technological effort: an analysis of their determinants in Brazilian firms", Journal of Industrial Economics, vol. 39, № 4, Wiley.

Cainelli, G., R. Evangelista y M. Savona (2006), "Innovation and economic performance in services: a firmlevel analysis", Cambridge Journal of Economics, vol. 30, № 3, Oxford University Press.

Carayannis, E., U. Varblane y T. Roolaht (2012), "Innovation systems in small catching-up economies. New perspectives on practice and policy", Innovation, Technology, and Knowledge Management, vol. 15, Springer.

Cohen, W. y S. Klepper (1996), "Firm size and the nature of innovation within industries: the case of process and product R\&D", The Review of Economics and Statistics, vol. 78, N2 2, Cambridge, Massachusetts, The MIT Press.

Comisión Consultiva para la Ciencia, Tecnología e Innovación (2012), "Nueva política e institucionalidad para dinamizar la CTI peruana" [en línea] http://franciscosagasti.com/descargas/eventos/ff-informe-comisionconsultiva-cti.pdf.

Comisión Europea (2011), "Policies in support of service innovation", Global Review of Innovation Policy Studies INNO-Grips, INNO-Grips Policy Brief, №3, Budapest, ICEG European Center.

CONCYTEC/INEI (Consejo Nacional de Ciencia, Tecnología e Innovación Tecnológica/Instituto Nacional de Estadística e Informática) (2005a), Encuesta Nacional de Ciencia, Tecnología e Innovación Tecnológica (ENCYT-04), Lima.

(2005b), "Análisis de la Encuesta Nacional de Ciencia, Tecnología e Innovación Tecnológica, 2004", Lima, inédito.

Crepon, B., E. Duguet y J. Mairesse (1998), "Research, innovation and productivity: an econometric analysis at the firm level", Economics of Innovation and New Technology, vol. 7, № 2, Taylor \& Francis.

Crespi, G. y P. Zúñiga (2010), "Innovation and productivity: evidence from six Latin American countries", IDB Working Paper Series, NN IDB-WP-218, Washington, D.C., Banco Interamericano de Desarrollo.

Crespi, G. y otros (2006), "Measuring and understanding productivity in UK market services", Oxford Review of Economic Policy, vol. 22, № 4, Oxford University Press.

26 Véase este resultado en Tello (2011). 
Crespi, G. y F. Peirano. (2007), "Measuring innovation in Latin America: what we did where we are and what we want to do", documento presentado en la Conference on Micro Evidence on Innovation in Developing Countries, Maastricht.

Dean, E. y K. Kunze (1992), "Productivity measurement in service industries", Z. Griliches (ed.), Output Measurement in the Service Sectors, Chicago, University of Chicago Press.

Evangelista, R. y M. Savona (2003), "Innovation, employment and skills in services: firm and sectoral evidence", Structural Change and Economic Dynamics, vol. 14, № 4, Amsterdam, Elsevier.

Gallouj, F. (2002), Innovation in the Service Economy: The New Wealth of Nations, Northampton, Edward Elgar Publishing.

Gallouj, F. y F. Djellal (2010), The Handbook of Innovation and Services, Northampton, Edward Elgar Publishing. (2008), Measuring and Improving Productivity in Services. Issues, Strategies and Challenges, Cheltenham, Edward Elgar Publishing.

Gallouj, F. y M. Savona (2009), "Innovation in services: a review of the debate and a research agenda", Journal of Evolutionary Economics, vol. 19, №2, Springer.

Gallouj, F. y O. Weinstein (1997), "Innovation in services", Research Policy, vol. 26, № 4-5, Amsterdam, Elsevier.

Garrido, C. (2009), "La innovación en los servicios: aspectos generales y los casos de los servicios de telecomunicaciones, turismo y bancario y bancario", Documento de Proyecto (LC/W.286), Santiago, Comisión Económica para América Latina y el Caribe (CEPAL).

Girma, S. y H. Görg (2007), "The role of the efficiency gap for spillovers from FDI: evidence from the UK electronics and engineering sectors", Open Economies Review, vol. 18, № 2, Springer.

Griliches, Z. (1992), Output Measurement in the Service Sectors, Chicago, The University of Chicago Press. (1979), "Issues in assessing the contribution of research and development to productivity growth", Bell Journal of Economics, vol. 10, № 1, The RAND Corporation.

Griliches, Z. y A. Pakes (1980), "Patents and R\&D at the firm level: a first look", NBER Working Paper, № 561 , Cambridge, Massachusetts, National Bureau of Economic Research.

Hahn, C. y C. Park (2010), "Direction of causality in innovation-exporting linkage: evidence on Korean manufacturing", ERIA Discussion Paper, № 2012-07.

Hall, B. (2011), "Innovation and productivity", NBER Working Paper, № 17178, Cambridge, Massachusetts, National Bureau of Economic Research.

Hanley, A. y J. Monreal-Pérez (2011), "Are newly exporting firms more innovative? Findings from matched Spanish innovators", Kiel Working Paper, № 1735, Kiel, Instituto de Economía Mundial.

INEI (Instituto Nacional de Estadística e Informática) (2005), "Encuesta económica anual 2005: estadística manufacturera", Lima.

Ito, K. (2011), "Sources of learning-by-exporting effects: does exporting promote innovation?", RIETI Discussion Papers Series, № 11066.

Kumar, N. y A. Aggarwal (2005), "Liberalization, outward orientation and in-house R\&D activity of multinational and local firms: a quantitative exploration for Indian manufacturing", Research Policy, vol. 34, $\mathrm{N}^{\circ} 4$, Amsterdam, Elsevier.

Kuramoto, J. (2008), "Propuesta de Agenda de Investigación para el Programa de Ciencia y Tecnología - FINCYT", Lima [en línea] http://www.innovateperu.gob.pe/fincyt/doc/CIES/Propuesta-Agenda-Investigacion.pdf. (2007), "Retos del Plan Nacional de Ciencia, Tecnología e Innovación Tecnológica para la Competitividad y Desarrollo Humano", inédito.

Kuramoto, J. y J. Díaz (2011), "Aportes para el gobierno peruano 2011-2016: Políticas de ciencia, tecnología e innovación”, Elecciones 2011 Perú: centrando el debate electoral, № 14, Lima.

- (2010), Evaluación de políticas de apoyo a la innovación en el Perú, Lima, Grupo de Análisis para el Desarrollo (GRADE).

Llisteri, J. y J. García-Alba (2008), "High-growth SMEs in Latin American emerging economies", Notas Técnicas, N ${ }^{\circ}$ IDB-TN-133, Washington, D.C., Banco Interamericano de Desarrollo.

Masso, J. y P. Vahter (2011), The Link Between Innovation and Productivity in Estonian Service Sector, Universidad de Tartu.

Mairesse, J. y P. Mohnen (2010), "Using innovations surveys for econometric analysis”, NBER Working Paper, NN 15857, Cambridge, Massachusetts, National Bureau of Economic Research.

Mairesse, J. y M. Sassenou (1991), "R\&D and productivity: a survey of econometric studies at the firm level", Science-Technology-Industry Review, № 8, París, Organización para la Cooperación y el Desarrollo Económicos (OCDE). 
Mohnen, P. y L.H. Röller (2005), "Complementarities in innovation policy", European Economic Review, vol. 49, N6, Amsterdam, Elsevier.

Mothe, C. y T.U. Nguyen Thi (2010), "The impact of non-technological innovation on technological innovation: do services differ from manufacturing? An empirical analysis of Luxembourg firms", CEPS /Instead Working Paper, N²010-01.

OCDE (Organización para la Cooperación y el Desarrollo Económicos) (2009), Innovation in Firms: A Microeconomic Perspective, París.

Peru Top Publications (2002-2007), Peru: The Top 10,000 Companies, Lima.

Raffo, J., S. Lhuillery y L. Miotti (2008), "Northern and Southern innovativity: a comparison across European and Latin American countries", European Journal of Development, vol. 20, № 2, Taylor \& Francis.

Rubalcaba, L., D. Gago y J. Gallego (2010), "On the differences between goods and services innovation", Journal of Innovation Economics \& Management, vol. 1, № 5.

Sagasti, F. (2011), En Busca del Tiempo Perdido: Ciencia, tecnología e innovación en el Perú, Lima, Foro Nacional Internacional.

SUNAT (Superintendencia Nacional de Aduanas y Administración Tributaria) (2012), Series estadísticas de exportaciones, Lima.

Syverson, C. (2011), "What determines productivity?", Journal of Economic Literature, vol. 49, № 2, Nashville, Tennessee, American Economic Association.

Tacsir, E. y otros (2011), "Innovation in services: the hard case for Latin America and the Caribbean", IDB Discussion Paper, NN IDB-DP-203, Washington, D.C., Banco Interamericano de Desarrollo.

Tello, M.D. (2012a), "Balance y agenda de investigación en tema de la inserción de la economía internacional", Balance y agenda de investigación en el Perú, Lima, Consorcio de Investigación Económica y Social (CIES). (2012b), "Productividad total factorial en el sector manufacturero: un análisis a nivel de firmas del Perú, 2002-2007", Economía, vol. 35, № 70, Lima, Pontificia Universidad Católica del Perú.

(2011), "Science and technology, ICT and profitability in the manufacturing sector in Peru", ICT in Latin America: A Micro-Data Analysis (LC/R.2172), M. Balboni, S. Rovira y S. Vergara (eds.), Santiago, Comisión Económica para América Latina y el Caribe (CEPAL).

(2010), Políticas de tecnologías de información y comunicación en el Perú, 1990-2010", Documentos de Trabajo, № 335, Lima, Pontificia Universidad Católica del Perú.

Tether, B.S. (2005), "Do services innovate (differently)? Insights from the European Innobarometer Survey", Industry and Innovation, vol. 12, N² 2, Taylor \& Francis.

Tybout, J., H. Katayama y S. Lu (2009), "Firm-level productivity studies: illusions and a solution”, International Journal of Industrial Organization, vol. 27, № 3, Amsterdam, Elsevier.

UNCTAD/CEPAL (Conferencia de las Naciones Unidas sobre Comercio y Desarrollo/Comisión Económica para América Latina y el Caribe) (2011), Science, Technology and Innovation Policy Review: Peru (UNCTAD/ DTL/STICT/2010/2), Ginebra. 